Recherches en didactique des langues et des cultures

Les cahiers de l'Acedle

11-2| 2014

Plurilinguisme(s) et entreprise : enjeux didactiques

\title{
Les contrats de travail en Italie et en France : un enjeu plurilingue pour les entreprises?
}

\section{Enrica Bracchi et Carolina Simoncini}

\section{OpenEdition}

\section{Journals}

Édition électronique

URL : http://journals.openedition.org/rdlc/1789

DOI : $10.4000 /$ rdlc. 1789

ISSN : 1958-5772

Éditeur

ACEDLE

Référence électronique

Enrica Bracchi et Carolina Simoncini, « Les contrats de travail en Italie et en France : un enjeu plurilingue pour les entreprises ?", Recherches en didactique des langues et des cultures [En ligne], 11-2 | 2014, mis en ligne le 07 juin 2014, consulté le 01 mai 2019. URL : http://

journals.openedition.org/rdlc/1789; DOI : 10.4000/rdlc.1789

Ce document a été généré automatiquement le 1 mai 2019.

\section{$(\mathbb{Q} \Theta \Theta$}

Recherches en didactique des langues et des cultures is licensed under a Creative Commons AttributionNonCommercial-NoDerivatives 4.0 International License 


\title{
Les contrats de travail en Italie et en France : un enjeu plurilingue pour les entreprises?
}

\author{
Enrica Bracchi et Carolina Simoncini
}

\section{Introduction}

1 La connaissance de la terminologie des contrats de travail dans la langue-culture des pays partenaires commerciaux permet une collaboration avantageuse et une plus grande transparence des rapports économiques, dans le respect des réglementations nationale, européenne ou internationale - régissant les rapports entre employeurs et salariés. Afin d'atteindre ce but, la maîtrise des termes d'une ou plusieurs languescultures, la connaissance du sens juridique des unités terminologiques (termes simples, syntagmes, acronymes...) ainsi que la prise en compte de la "place bien précise" (Penfornis, 2001) que celles-ci occupent dans un texte technique donné sont indispensables. En effet, bien que les langues-cultures et langues-cultures juridiques de travail soient très proches comme dans le cas de l'italien et du français, parfois ces unités terminologiques spécialisées ne sont pas si transparentes et évidentes qu'on pourrait le croire.

Dans la présente contribution, nous nous proposons donc d'aborder la question de l'enseignement-apprentissage du lexique juridique propre aux contrats de travail à des étudiants non-juristes, au service d'une approche plurilingue en entreprise. Pour cela nous mènerons une réflexion en parallèle entre langue-culture et langue-culture juridique, dans la perspective d'un plurilinguisme fonctionnel. 


\section{L'enseignement-apprentissage de la terminologie des contrats à des apprenants non-juristes dans la perspective d'un plurilinguisme fonctionnel}

Au cours de l'année universitaire 2012-2013, nous avons dispensé le cours d'italien "Communication professionnelle orale", destiné aux étudiants de Licence 3 L.E.A. de l'Université Rennes 2, options "Traduction et communication multilingue" et "Langues et commerce international". Bien que le droit - et plus précisément le droit de l'entreprise - ne soit que l'une des matières d'application parmi beaucoup d'autres (commerce international, négociation internationale, marketing...) qui sont suivies par les étudiants en troisième année de Licence, nous avons fait le choix de présenter un cours d'italien appliqué au droit. En effet, même si les apprenants ne sont pas des juristes, dans la perspective d'un futur stage et/ou travail ils pourront être amenés à être confrontés à des textes juridiques et à la terminologie correspondante.

Dans le cadre de ce cours, la maîtrise des mots et des termes techniques est donc cruciale.

C'est pour cette raison que, tout d'abord, les étudiants ont été amenés à comprendre l'importance de la terminologie qui s'intéresse à l'étude des termes (également appelés "unités terminologiques"), qui sont des unités linguistiques - mais également de communication - dont les caractéristiques s'apparentent à celles des mots. Dans la première partie du cours nous avons proposé un encadrement théorique aux étudiants pour leur démontrer comment les mots n'acquièrent le statut de terme que "dans la mesure où [il] se situe dans la structure d'un domaine de spécialité" (Cabré, [1992] 1998 : 194), propre à une science, à une technique ou à une activité restreinte. Lorsqu'il est défini dans un domaine spécialisé, dans un contexte concret et d'usage bien déterminé, le terme ne devrait avoir qu'un seul signifié, univoque, monoréférentiel et donc non ambigu. La première étape de notre cours a donc été caractérisée par une réflexion sur la terminologie afin que notre public comprenne que celle-ci se sert des termes pour bien circonscrire des notions, pour dénommer et pour appeler un objet avec son nom précis, dans un souci de normalisation et de standardisation des termes. Une attention plus particulière a été portée sur la terminologie juridique, qui constitue le cœur de notre cours. Elle doit in primis satisfaire des besoins propres au système juridique et à la langueculture d'un pays donné. L'étude et l'analyse de la terminologie juridique demandent en effet des compétences culturo-juridiques, et non seulement des compétences linguistiques nécessaires à la communication spécialisée. Pour ce qui est des besoins, ils devraient être satisfaits tant sur le plan national que sur le plan européen et international, dans le but de faciliter la communication entre des spécialistes issus d'horizons géographiques et scientifiques différents. Dans la mesure où une rigueur scientifique s'avère indispensable lors de l'analyse de la langue, la terminologie juridique a ses propres règles, tout en puisant dans la théorie générale de la linguistique ou de la syntaxe, et dans d'autres disciplines non linguistiques, ce qui lui permet d'aborder des sujets qui ne relèvent pas seulement du droit, car ils concernent directement d'autres sphères du savoir, l'"empreinte culturelle" (Gémar, 2002 ; Terral, 2004) étant omniprésente dans le vocabulaire du droit.

5 Dans le cas spécifique des approches didactiques de la terminologie du droit, nous exploitons des synergies et nous créons des liens entre des compétences (linguistiques et 
non-linguistiques) (Carvalho, 2010), selon une "approche plurielle des langues et des cultures" (Candelier et al., $2007: 3$ ), à savoir "des approches didactiques [mettant] en ceuvre des activités d'enseignement-apprentissage qui impliquent à la fois plusieurs (= plus d'une) variétés linguistiques et culturelles" (Candelier et al., $2007: 3$ ). Grâce à notre expérience, nous avons néanmoins pu constater qu'il ne s'agit pas seulement de la mise en œuvre d'activités impliquant plusieurs variétés linguistiques et culturelles, mais aussi d'une ou plusieurs "variétés juridiques", à savoir branches et sous-branches du droit. En effet, "qui veut comprendre les mots du droit n'a guère d'autre choix que d'appréhender le système juridique dans lequel ces mots s'insèrent et dont ils sont indissociables" (Penfornis, 2001). L'enseignementapprentissage de la terminologie juridique de différents pays demande également une approche d'un enseignement et d'un apprentissage "combinés et coordonnés" (Zappatore, 2007: 21 in Sauer, Saudan 2008: 5) des langues-cultures et des languescultures juridiques.

Ce cadre théorique avait pour finalité de faire comprendre aux étudiants l'importance de savoir communiquer langagièrement autour de la question des contrats de travail, dans un contexte économique et juridique donné, pour la réalisation d'activités variées, pouvant relever de la réception, de la production, de l'interaction et de la médiation. L'échange avec le groupe-classe a ainsi porté sur les différences juridico-terminologiques du langage français (de France) et italien (d'Italie) des contrats, et dans le spécifique sur le degré de protection accordée (ou non) aux salariés et aux travailleurs indépendants, dans les deux pays de travail.

\section{Les contrats de travail : un enjeu plurilingue pour les entreprises?}

7 L'analyse des termes spécialisés des contrats de travail nous a en effet permis de mener une réflexion langue-culture-droit, en nous concentrant sur les contrastes qui découlent d'une comparaison entre des concepts juridico-culturels propres à l'Italie et à la France. Dans le cas spécifique de ce cours, nous avons explicitement évité de faire une analyse d'un contrat-type car cette approche aurait été trop juridique et elle aurait demandé davantage de connaissances et de compétences de la part des apprenants en termes de contrats italiens et français.

8 Nous avons préféré proposer aux étudiants d'analyser de la réglementation générale portant sur les contrats de travail. Pour cela, nous avons divisé la classe en groupes de quatre personnes. Chaque groupe devait monter sa propre entreprise fictive en choisissant, parmi les contrats prévus par le droit italien et le droit français, les contrats qu'ils considéraient comme les plus appropriés au type d'entreprise choisi, tout en motivant leurs choix. C'est grâce à cette démarche que les apprenants se sont rendu compte de l'existence de profondes différences entre les deux systèmes. Lors de l'échange enseignant/groupe classe en cours, nous avons donc pu identifier avec les étudiants les éléments terminologiques importants pour une réflexion plurilingue, qui ont fait surgir des interrogations autour du sens et de la portée juridique des termes employés, lors du passage d'une langue-culture juridique à l'autre.

9 Une première remarque sur le syntagme contratto di lavoro/contrat de travail s'impose. Ensuite, l'analyse des répercussions (flexibilité vs garanties des travailleurs) des différentes typologies de contrats de travail sur le statut du salarié nous a semblé 
importante afin de dégager des aspects historico-civilisationnels propres à l'un ou à l'autre pays. Il nous est également paru essentiel de souligner que la présence d'anglicismes dans la terminologie propre aux contrats de travail en Italie peut créer des malentendus et, pour terminer, nous avons identifié, avec les étudiants, des termes que nous avons définis comme étant "à forte connotation culturelle".

\section{Contratto $=$ contrat $?$}

Le premier problème qui a émergé dans le cadre du cours dédié aux contrats de travail a été justement la compréhension du syntagme contrat de travail, toujours traduit en italien par contratto di lavoro.

11 Le terme contratto/contrat, en Italie comme en France, renvoie à une "convention faisant naître une ou plusieurs obligations ou bien créant ou transférant un droit réel" (Guinchard, Montagnier, 2007). Toutefois, lorsque ce même terme est utilisé pour constituer le syntagme contratto di lavoro/contrat de travail, il existe une différence juridique entre les deux pays. Lors d'un débat organisé pendant le cours, les étudiants ont pu remarquer qu'en France, l'expression "contrat de travail" se limite au rapport subordonné entre employeur et travailleur, alors que dans le système juridique italien, la même expression "contratto di lavoro" peut se référer à la fois au rapport subordonné et au rapport entre travailleurs autonomes (Hascoët, 2010: 23). À la traduction littérale de l'italien vers le français de contratto di lavoro il sera donc préférable d'ajouter les adjectifs "subordonné" ou "indépendant" (Hascoët, 2010: 23), selon le contexte dans lequel le syntagme est utilisé. Les apprenants se trouvent ici confrontés à une première exception culturelle et juridique italienne, bien que le syntagme contratto di lavoro soit le plus souvent traduit par "contrat de travail" et vice-versa, sans interrogation aucune sur la portée et l'implication juridiques de cette suite de mots, et cela notamment au niveau des obligations qui en découlent et des retombées juridiques possibles dues à une mauvaise utilisation et/ou transposition des concepts d'une sphère lexico-culturelle à une autre.

\section{Typologies de contrats de travail en Italie et en France : flexibilité vs garanties pour le travailleur}

12 Les intitulés des contrats de travail en Italie et en France dévoilent la difficulté, pour des étudiants non-juristes, de comprendre le type de relation de travail qui existe derrière ces formules très différentes entre les deux pays. Pour permettre aux apprenants d'atteindre une véritable connaissance du système des contrats de travail en Italie, il a fallu passer par l'analyse du modèle français. Les étudiants ont tout d'abord analysé le fonctionnement des contrats de travail français: des conventions qu'ils connaissaient mieux grâce à leurs propres expériences de travail. À partir de ces connaissances acquises précédemment, nous avons pu leur faire remarquer les différences évidentes avec le système italien, surtout par rapport à la "sûreté" des contrats français opposée à la "précarité" des contrats italiens.

Dans cette phase de comparaison, nous avons tout d'abord remarqué qu'il existe plusieurs typologies de contrats de travail aux caractéristiques propres, selon les deux pays que nous avons retenus pour cette étude. Le droit français prévoit cinq principaux types de contrats (cf. Annexe) et, comme les intitulés des conventions entre employeur et salarié l'indiquent, dans les contrats de travail français l'accent est mis sur la durée du temps de 
travail. Cette durée peut se référer d'une part à la collaboration entre les parties ("durée indéterminée" ou "durée déterminée") et d'autre part à l'amplitude horaire de travail du salarié ("à temps plein (ou complet)" et "à temps partiel").

Le contrat à durée indéterminée (à temps plein ou partiel) est le contrat le plus stable et le plus sûr pour la vie d'un travailleur salarié. À ce propos, il est important de souligner qu'encore aujourd'hui le Ministère français du Travail, de l'Emploi de la Formation professionnelle et du Dialogue social définit le contrat à durée indéterminée comme "la forme normale et générale de la relation de travail" (Le contrat de travail à durée indéterminée [CDI]). Cette forme de convention reste - et cela malgré le débat sur la flexibilité du travail - le point de repère pour les employeurs français.

En effet, en France, malgré le fait que de nouveaux contrats aient été intégrés dans la législation, l'employeur n'y a recours que dans des cas particuliers, sans modifier les garanties prévues pour les travailleurs par les contrats "de base". À ce propos, le droit du travail français est très clair et ne provoque aucun malentendu ni sur le plan terminologique ni sur le plan des garanties pour le travailleur salarié. La loi introduit des contrats définis comme "précaires" et qui, comme la définition de l'adjectif l'indique "ne peu[ven]t garantir la durée, la solidité, la stabilité" (CNTRL - Centre National de Ressources Textuelles et Lexicales). Il s'agit, par exemple, du "contrat de travail temporaire" qui ne peut être conclu que pour l'exécution d'une tâche précise et temporaire nommée "mission", et seulement dans les cas prévus par la loi. Un autre exemple de contrat précaire est le "contrat de travail saisonnier" qui se caractérise par - nous citons - " l'exécution de tâches normalement appelées à se répéter chaque année, à des dates à peu près fixées " (Définition du travail saisonnier).

En ce qui concerne l'Italie, cinq principaux types de contrat sont prévus par le système juridique, comme pour la France (cf. Annexe). Au niveau des intitulés des contrats, les étudiants ont remarqué que la terminologie italienne est très proche de la terminologie française ${ }^{1}$. Toutefois, grâce à l'approche choisie pour aborder les contrats de travail (réflexion langue-culture-droit), au fil du temps, les apprenants ont pris conscience du fait que la situation en Italie est radicalement différente par rapport à la France : ces contrats "de base" qui garantissaient un certain niveau de stabilité et de protection pour les travailleurs ont été remplacés par d'autres conventions. En effet, depuis les années 1990, on assiste à une explosion des emplois précaires et $70 \%$ des embauches sont réalisées grâce aux contrats de travail subordonné à durée déterminée, comme par exemple les contratti di collaborazione coordinata e continuativa (traduit littéralement par "contrats de collaboration coordonnée et continue"), également connus sous le sigle co.co.co. Selon cette convention, le salarié s'engage à fournir une œuvre ou un résultat final sans aucune obligation de la part de l'employeur, en termes de modalités d'accomplissement de la tâche et de temps de travail. Les contratti di collaborazione coordinata e continuativa n'ont aucune correspondance dans le droit français (Hascoët, $2010: 23)$.

De plus, au cours du deuxième gouvernement Berlusconi (juin 2001 - avril 2005) et à la suite de l'entrée en vigueur de la loi n. 2003-30 de réforme du travail dite "réforme Biagi" - nommée ainsi en l'honneur de l'expert en droit du travail Marco Biagi, assassiné en 2002 par les nouvelles Brigades Rouges - le nombre de contrats s'est davantage démultiplié. Pendant cette période, l'élément qui a contribué à créer une certaine confusion dans le lexique et dans les appellations du droit de travail est le terme flessibilità/flexibilité, ainsi que sa définition. Au début des années 2000, le discours 
politique général annonçait que la flexibilité allait être la seule solution possible pour pallier à la crise économique qui s'amorçait. La "réforme Biagi" a introduit de nouvelles formes de contrat de travail qui, toutefois, n'ont pas été définies explicitement ni classées comme "précaires", même si elles l'étaient selon la législation. De cette façon, les (futurs) travailleurs ont été confrontés à de nouveaux contrats précaires de travail, parmi lesquels le contratto di somministrazione di lavoro, que nous pouvons traduire littéralement par "contrat d'administration — dans le sens de "donner", "fournir", "faire bénéficier" — du travail". Aux termes de ce contrat, l'employeur s'engage à offrir à un autre employeur la main-d'œuvre de ses employés, pour une période de temps limitée. Le contratto di lavoro ripartito (connu également sous le terme anglo-saxon de job sharing)/"contrat de travail partagé", constitue quant à lui une variante du contrat à temps partiel. Une unité de travail, c'est-à-dire l'équivalent des heures de travail normalement attribuées à un seul travailleur, est ainsi partagée entre deux travailleurs qui sont chacun leur tour responsables de l'exécution d'une tâche donnée.

Il existe ensuite le contratto di lavoro a chiamata o intermittente/"contrat de travail sur appel ou intermittent", ou job on call. Le travailleur donne sa disponibilité à l'employeur pour une période de temps limitée. L'employeur pourra appeler ou non le travailleur qui sera rémunéré d'une part en fonction du travail effectué et d'autre part en fonction de la disponibilité proposée à l'employeur.

19 Les étudiants ont ainsi pu comprendre que les contrats italiens de base, proches des modèles français auxquels ils étaient habitués, ont été complétement mis à l'écart pour faire surgir la culture du travail précaire, contrairement à la France qui a essayé de garder une certaine forme de stabilité.

\section{Anglicismes}

Une difficulté supplémentaire de compréhension des contrats de travail italiens a été, pour les étudiants, la présence d'appellations anglo-saxonnes, y compris dans le langage juridique officiel, associées à des expressions italiennes, comme nous venons de le voir au sujet du contratto di lavoro ripartito/job on sharing et du contratto di lavoro a chiamata o intermittente/job on call. Bien que très fréquent, le recours à ces emprunts lexicaux nous semble être superflu (Adamo, Della Valle, 2009 : 27), comme c'est d' ailleurs très souvent le cas pour les termes anglais utilisés alors qu'un terme italien existe déjà. Ce choix langagier pourrait créer - voire accroître - une certaine confusion au sujet des différentes typologies de contrats de travail. Cette même confusion nous semble être également accentuée par l'existence d'un trop grand nombre de parasynonymes, phénomène probablement dû à un manque de normalisation terminologique. Pour illustrer ce phénomène, nous faisons référence au syntagme contratto di lavoro a chiamata, nommé également - y compris dans les textes juridiques - contratto di lavoro intermittente ou job on call.

21 En plus des anglicismes, il convient de souligner la présence de sigles. En italien, la siglaison est un procédé linguistique beaucoup moins usité qu'en français. Ainsi, le choix de désigner - y compris dans les textes officiels - le contratto di collaborazione coordinata e continuativa par co.co.co ou le contratto di lavoro a progetto/"contrat de travail sur projet" par co.co.pro nous semble d'une part augmenter la confusion et d'autre part camoufler le contenu de ces contrats. 
Les différentes typologies de contrats de travail que nous venons d'analyser, en apparence, ont été conçues pour protéger les travailleurs les plus faibles comme, par exemple, les jeunes ou les travailleurs de plus de 55 ans, un âge très avancé pour réintégrer le marché du travail. Toutefois, dans la réalité, ces modèles se sont substitués aux contrats de travail "traditionnels". Par conséquent, aujourd'hui, à la différence de la France, le contrat à durée indéterminée n'est presque plus appliqué en Italie. La plupart des travailleurs italiens sont ainsi embauchés par le biais de contrats flexibles, voire précaires. Même si la "réforme Biagi" a voulu mettre l'accent sur la flexibilité en donnant à celle-ci une connotation positive, nous remarquons, sur le fond, que la flexibilité italienne est bel et bien devenue un véritable parasynonyme de précarité. Par ailleurs, les adjectifs et les compléments employés pour nommer les différents contrats nous semblent souligner cette "flexibilité-précarité". Le (jeune) travailleur s'engage, par exemple, à rester à la disposition de l'employeur qui l'appellera - à ce propos nous renvoyons à l'expression a chiamata, calque de l'expression anglo-saxonne on-call contract (Treccani.it - Neologismi, 2008) - et cela en fonction des besoins de l'employeur. Le (jeune) travailleur offrira également ses services à des intervalles plus ou moins réguliers, tout comme un intermittent, terme français sur lequel l'adjectif substantivé italien intermittente a été calqué (Treccani.it - Neologismi, 2008). La situation de précarité de l'emploi nous semble être également soulignée par une autre expression et notamment par le syntagme a progetto, qui renvoie au fait que le rapport de collaboration nait sur la base d'un objectif à atteindre, indépendamment du nombre d'heures nécessaires pour y parvenir (Treccani.it - Neologismi, 2008). En outre, l'adjectif occasionale met l'accent sur l'une des caractéristiques de ce contrat : il peut être souscrit par les parties, seulement si l'"occasion", la "circonstance" se produit. Enfin, l'adjectif accessorio renvoie au fait que l'activité vient s'ajouter à une situation déjà existante, par exemple dans le cas d'une activité menée dans le cadre d'une mission non réglementée, d'une tâche accomplie "au noir" (Lavoro accessorio).

\section{Termes à forte connotation culturelle}

Enfin, dans le cadre du cours de "Communication professionnelle orale" nous avons amené les apprenants à réfléchir sur la profonde différence de traitement des travailleurs en Italie et en France, avec une attention particulière sur les garanties prévues, ou non, pour les travailleurs. C'est ainsi que dans le cadre d'un exercice de pratique orale, nous avons invité les étudiants à citer des termes - en français ou en italien — liés au monde du travail et des contrats, pour vérifier d'une part s'il existait un équivalent dans l'autre langue et d'autre part si ces termes renvoyaient à cette différence de traitement identifiée auparavant. Parmi les termes évoqués par les apprenants "SMIC", "RTT" et "congés payés" ont retenu notre attention. Il s'agit en effet de termes - et plus précisément de concepts - pour lesquels il n'existe aucune correspondance dans la législation italienne en matière de contrats de travail. Plus précisément, le "SMIC" et les "jours de RTT" ne sont pas prévus par le système juridique italien. Il n'est pas rare que les jeunes travailleurs italiens perçoivent une rémunération de seulement 200-300 euros par mois et, dans la plupart des cas, aucune compensation pécuniaire n'est prévue pour les stagiaires. Pour ce qui est de l'octroi des "jours de RTT", le droit italien ne prévoit aucune forme de réduction du temps de travail pour atteindre le temps de travail légal en France de 35 heures, contre 48 heures en Italie. Quant aux "congés payés", l'article L3141-3 du 
Code du travail français stipule que "le salarié a droit à un congé de deux jours et demi ouvrables ${ }^{2}$ par mois de travail effectif chez le même employeur" et donc à 25 jours de "congés payés" (vacances dans le lexique commun) par an, alors qu'en Italie les travailleurs bénéficient de quatre semaines légales de ferie (Renour, 2011), en plus des 24 heures consécutives de repos hebdomadaire (De Matteis et al., 2011). Si en italien, le terme utilisé pour traduire le syntagme français "congés payés" est ferie - terme qui renvoie, de manière générale, à la période de repos estival et par conséquent de vacances - lorsqu'il existe une indication de la raison qui justifie cette "dispense de travail pour un temps de repos ou une autre convenance" (Cornu, 2007), c'est le terme congedo qui est utilisé, comme dans les cas de congedo per maternità/"congé de maternité", de congedo per paternità /"congé de paternité", de congedo per malattia/"congé de maladie"...

Le syntagme cassa integrazione - que nous pourrions traduire littéralement par "caisse d'intégration" et qui correspondrait à une sorte de "mise en chômage technique" - est revenu plusieurs fois. Si ce terme en tant que séquence de signes est connu par les étudiants, nous avons pu constater que son sens juridique ne leur était pas clair. En effet, le plus souvent, l'apprenant français perçoit le système de cassa integrazione comme un système d'aides mis en place par l'État italien. Dans ce syntagme, le substantif adjectivé integrazione/intégration est - peut-être délibérément? - trompeur car il renvoie aux verbes "intégrer", "assimiler" et il serait donc justifié de penser à une mesure de l'État visant à insérer à nouveau une personne dans le monde du travail. Cette ambiguïté est davantage renforcée par le fait que l'expression juridique exacte est cassa integrazione guadagni/"caisse d'intégration des gains", le terme guadagni étant également trompeur car l'apprenant pourrait penser à une caisse qui contient de l'argent disponible pour les travailleurs. Dans la réalité du contexte de travail italien, la cassa integrazione est, en revanche, la période pendant laquelle les salariés sont soumis à une réduction de leur temps de travail et de leur salaire, avant le licenciement quasiment certain. La cassa integrazione est ainsi une sorte d'"antichambre" du licenciement et non pas une sorte d'"antichambre" en vue d'une nouvelle embauche.

Un autre terme cité par les étudiants et qui peut induire en erreur un apprenant francophone est mobilità/"mobilité". Si en français le terme "mobilité" - et plus précisément "mobilité professionnelle" - réfère à l'"adaptation aux changements dans la qualification des emplois, rendue nécessaire par le progrès technique et les modifications des structures économiques" (Cornu, 2007), en italien en revanche, le terme mobilità renvoie à une institution de protection du chômage qui est en partie reliée au système de cassa integrazione. En Italie, en plus d'une indemnité de chômage comme en France, il est prévu une "indemnité de mobilité" 3 . Celle-ci permet à l'entreprise de gérer le personnel supplémentaire en l'affectant dans une autre entreprise œuvrant sur le même territoire. Cette indemnité n'est versée qu'aux travailleurs licenciés par des entreprises obligées de réduire leurs effectifs à cause d'une crise économique, d'un manque de travail, d'une fermeture de bureaux...

\section{Conclusion}

26 À travers l'analyse des spécificités de quelques termes propres aux contrats de travail, les apprenants ont pu prendre conscience des éléments spécifiques aux deux languescultures dans lesquelles ils seront amenés à communiquer professionnellement, dans le cadre d'un stage ou d'un emploi futur. Il est également ressorti de manière très claire que, 
si auparavant, dans les deux pays, le travail était le "lieu symbole d'une stabilité construite autour de l'idée du "statut" du salarié" (Perulli in Hascoët, 2010: 7), aujourd'hui cette stabilité serait "fluide, fragile" (Perulli in Hascoët, $2010: 7$ ) et il est désormais préférable de parler de précarité plutôt que de stabilité.

C'est au vu des différents éléments terminologiques, culturels et juridiques mis en évidence grâce aux activités mises en place (conversation, débat, simulation) lors de notre cours que les apprenants ont pris conscience de la nécessité de la maîtrise de la langue, de la culture et du droit de l'Autre-partenaire commercial. Il nous semble ainsi que dans la sphère des relations entre employeurs et employés une maîtrise plurilingue, pluriculturelle et plurijuridique des contrats de travail est indispensable pour améliorer les transferts de compétences dans un contexte professionnel donné.

\section{BIBLIOGRAPHIE}

Adamo, G., Della Valle, V. (2009). Le parole del lessico italiano, Roma : Carocci.

Cabré, M. T.. ([1992] 1998). La terminologie. Théorie, méthode et applications. Ottawa/Paris : Les Presses Universitaires de l'Université de Ottawa/Armand Collin.

Cornu, G. (Publié sous la direction de) (2007). Vocabulaire juridique, Paris : PUF.

Guinchard S., Montagnier G. (Sous la direction de) (2007). Lexique des termes juridiques, Paris : Dalloz, $16^{\text {ème }}$ édition.

Hascoët, M. (2010). Le "contrat de travail" précaire en droit italien. Droit comparé italien et français. Aixen-Provence : Presses Universitaires d'Aix-Marseille.

Mounin George (dir.) ([1974] 2000). Dictionnaire de la linguistique, Paris : Quadrige/PUF

Candelier, M. (coordinateur), Camilleri-Grima A., Castellotti V., J.-F. de Pietro, Lörincz I., Meissner F.-J., Schröder-Sura A., Noguerol A. (2007). CARAP - Cadre de Référence pour les Approches Plurielles des Langues et des Cultures. Centre Européen pour les Langues Vivantes de Graz. http:// archive.ecml.at/mtp2/ALC/pdf/CARAP_F.pdf.

Carvalho, C. (2010). "Enseigner la langue du droit comme langue étrangère : cas du français juridique destiné à des étudiants espagnols". La culture de l'autre : l'enseignement des langues à l'Université - Actes. La Clé des Langues (Lyon : ENS LYON/DGESCO). ISSN 2107-7029. http:// cle.ens-lyon.fr/espagnol/enseigner-la-langue-du-droit-comme-langue-etrangere-cas-dufrancais-juridique-destine-a-des-etudiants-espagnols-91348.kjsp.

De Matteis, A., Accardo, P., Mammone, G. (2011). Présentation du droit du travail de l'Italie. http:// www.ilo.org/ifpdial/information-resources/national-labour-law-profiles/WCMS_158863/lang-fr/index.htm\#P3_64.

Gémar, J.-Cl. (2002). "Le plus et le moins-disant culturel du texte juridique. Langue, culture et équivalence". Meta : journal des traducteurs/Meta : Translators' Journal, juin 2002, vol. 47, n 2. http://www.erudit.org/revue/meta/2002/v47/n2/008006ar.pdf 
Penfornis, J.L. (2001). "Enseigner le français juridique, un langage de spécialité. Le droit, ce sont aussi des mots". Synergies pays riverains de la Baltique, $\mathrm{n}^{\circ}$ 1. pp. 129-132. http://gerflint.fr/Base/ Baltique1/fjuridique.pdf.

Renour, F. (2011). "Congés payés et jours fériés : le classement européen". Disponible sur : http:// www.journaldunet.com/management/vie-personnelle/conges-payes-et-jours-feries-parpays.shtml.

Sauer E., Saudan V. (2008). Aspects d'une didactique du plurilinguisme - Propositions terminologiques. Passpartout - Fremdsprachen an derVolksschule. http://clil-lote-go.ecml.at/LinkClick.aspx?

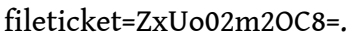

Terral F. (2004). "L’empreinte culturelle des termes juridiques". Meta : journal des traducteurs/ Meta : Translators' Journal, décembre 2004, vol. 49, n 4. http://www.erudit.org/revue/meta/2004/ v49/n4/009787ar.pdf.

\section{Sitographie}

Auto-entrepreneur ou salarié. Disponible sur : http://tarn.franceolympique.com/tarn/fichiers/File/ Lettre_electronique/2012/Juillet_2012/7_autoentrepreneur_et_salarie.pdf.

CNTRL - Centre National de Ressources Textuelles et Lexicales, entrée "précaire". http:// www.cnrtl.fr/definition/precaire.

Définition du travail saisonnier. http://www.pole-emploi.fr/actualites/definition-du-travailsaisonnier-@/suarticle.jspz?id=76764.

Lavoro accessorio. http://www.cliclavoro.gov.it/NormeContratti/Contratti/Pagine/Contratto-dilavoro-accessorio.aspx.

Le contrat de travail à durée indéterminée (CDI). http://travail-emploi.gouv.fr/informationspratiques,89/les-fiches-pratiques-du-droit-du,91/contrats,109/le-contrat-de-travail-aduree,3932.html.

Le contrat de travail temporaire. http://travail-emploi.gouv.fr/informations-pratiques,89/fichespratiques,91/contrats,109/le-contrat-de-travail-temporaire,982.html.

Services pour l'Emploi, Travail, Éducation et Formation en Italie - Les tutelles prévues pour les sans emplois .http://transalp.lavorosenzafrontiere.org/contenuti_fr/italia/tutelles_sans_emplois.htm.

Treccani.it - L'Enciclopedia italiana - Vocabolario - Neologismi (2008). http://www.treccani.it/ magazine/lingua_italiana/neologismi/searchNeologismi.jsp.

\section{ANNEXES}

\section{Les contrats de travail en France et en Italie}

\section{France :}

- contrat de travail à durée indéterminée à temps plein ;

- contrat de travail à durée indéterminée à temps partiel ;

- contrat de travail à durée déterminée à temps plein ;

- contrat de travail à durée déterminée à temps partiel ; 
- contrat de travail de formation en alternance.

Italie :

- contratto di lavoro a tempo indeterminato/contrat à durée indéterminée ;

- contratto di lavoro a tempo determinato/contrat à durée déterminée ;

- contratto di inserimento/contrat d'insertion ;

- contratto di apprendistato/contrat d'apprentissage ;

- contratto a tempo parziale/contrat à temps partiel ;

À la suite de la "réforme Biagi" (loi n. 2003-30) :

- contratti di collaborazione coordinata e continuativa (co.co.co.)/"contrats de collaboration coordonnée et continue"4 ;

- contratto di somministrazione di lavoro/"contrat d'administration du travail" [ancien contratto per prestazioni di lavoro temporaneo/"contrat pour des prestations de travail temporaire"] ;

- contratto di lavoro a chiamata ou contratto di lavoro intermittente ou job on call/"contrat de travail sur appel ou intermittent" ;

- contratto di lavoro ripartito ou job on sharing/"contrat de travail partagé" ;

- contratto di lavoro a progetto (co.co.pro.)/"contrat de travail sur projet" ;

- contratto di lavoro occasionale/"contrat de travail occasionnel" ;

- contratto di lavoro accessorioi/"contrat de travail accessoire".

\section{NOTES}

1. Contratto di lavoro a tempo indeterminato/contrat à durée indéterminée; contratto di lavoro a tempo determinato/contrat à durée déterminée; contratto di inserimento/contrat d'insertion; contratto di apprendistato/contrat d'apprentissage; contratto a tempo parziale/contrat à temps partiel.

2. Les jours "ouvrables" sont les jours de repos hebdomadaire (généralement le dimanche) et les jours fériés légaux et chômés dans l'entreprise. Les jours "ouvrés", en revanche, sont les jours normalement travaillés dans l'entreprise.

3. Pour la traduction cf., entre autres, Services pour l'Emploi, Travail, Éducation et Formation en Italie Les tutelles prévues pour les sans emplois. http://transalp.lavorosenzafrontiere.org/contenuti_fr/ italia/tutelles_sans_emplois.htm.

4. Nous indiquons, entre guillemets, les traductions littérales des syntagmes italiens.

\section{RÉSUMÉS}

La connaissance du lexique des contrats de travail dans la langue-culture des pays partenaires commerciaux peut permettre une collaboration avantageuse et une plus grande transparence des rapports économiques.

Dans la présente contribution, nous nous proposons d'aborder la question de l'enseignementapprentissage des termes propres aux contrats entre employeur et salariés, dans la perspective d'un plurilinguisme fonctionnel. Nous avons expérimenté un cours de ce type dans le cadre du 
cours de communication professionnelle italienne (filière L.E.A.), destiné à des étudiants francophones italianistes.

Après une étude des spécificités des contrats de travail italiens, les étudiants ont été invités à mener une comparaison entre les modèles des contrats de travail français et italiens dans le but d'identifier les différences sur le plan de la langue-culture et de la langue-culture juridique et de pouvoir ainsi correctement utiliser les termes italiens, dans la perspective d'un futur travail en Italie.

The knowledge of the vocabulary of labour contracts in the language/culture of countries which are commercial partners is an important skill when one aims to obtain fruitful collaborations and a better transparency of economic relations.

In this paper, we address the question of teaching/learning of terms which are related to contracts between the employer and the employee, in the perspective of functional multilingualism. We have experienced teaching this topic in the framework of the course of Italian professional communication (L.E.A. classes), for French students.

After a survey of some special characteristics of Italian labour contracts, the students have been asked to make a comparison between the models of French and Italian labour contracts, in order to identify the differences of language/culture and juridical language/culture. This work helps the students to use the Italian terms properly, in the perspective of a future job in Italy.

\section{INDEX}

Mots-clés : contrats de travail, langue-culture juridique, plurilinguisme fonctionnel, terminologie juridique

Keywords : functional plurilingualism, labour contracts, legal language/culture, legal terminology

\section{AUTEURS}

\section{ENRICA BRACCHI}

Enrica Bracchi est Maître de conférences à l'Université de Nantes et elle est rattachée au laboratoire CRINI (Centre de Recherches sur les Identités Nationales et l'Interculturalité). Ses travaux de recherche sont orientés autour d'un axe pluridisciplinaire (langue-droit-civilisation), tout en ayant comme présupposés théoriques l'évolution de la réglementation, de la terminologie et des mœurs, dans l'Italie contemporaine. Elle élargit également sa réflexion à d'autres languescultures européennes, dans une perspective plurilingue et pluriculturelle et elle s'intéresse à l'enseignement-apprentissage des langues-cultures sur des objectifs spécifiques et dans la mise en place de nouveaux dispositifs d'enseignement.

Courriel : enrica.bracchi@univ-nantes.fr

Adresse : CRINI (Centre de Recherches sur les Identités Nationales et l'Interculturalité) Université de Nantes - Faculté de Langues et Cultures Étrangères - Chemin de la Censive du Tertre - BP 81227 - 44312 Nantes cedex, France.

\section{CAROLINA SIMONCINI}

Carolina Simoncini est docteur en droit italien. Elle est actuellement lectrice à l'Université Rennes 2 et chargée de cours à l'Université de Nantes. Elle est rattachée au CRINI (Centre de Recherches sur les Identités Nationales et l'Interculturalité). 
Courriel : carolina.simoncini@univ-rennes2.fr

Adresse : Place du Recteur Henri le Moal. 35043, Rennes, France. 